\title{
Spacetime states and covariant quantum theory
}

\author{
Michael Reisenberger ${ }^{a b}$ and Carlo Rovelli ${ }^{a c}$ \\ ${ }^{a}$ Centre de Physique Théorique, Luminy, F-13288 Marseille, EU \\ ${ }^{b}$ Physics Department, Pittsburgh University, PA-15260, USA \\ ${ }^{c}$ Facultad de Ciencias, Universidad de la República, Iguá 4225, Uruguay
}

(July 16, 2018)

\begin{abstract}
In it's usual presentation, classical mechanics appears to give time a very special role. But it is well known that mechanics can be formulated so as to treat the time variable on the same footing as the other variables in the extended configuration space. Such covariant formulations are natural for relativistic gravitational systems, where general covariance conflicts with the notion of a preferred physical-time variable. The standard presentation of quantum mechanics, in turns, gives again time a very special role, raising well known difficulties for quantum gravity. Is there a covariant form of (canonical) quantum mechanics?

We observe that the preferred role of time in quantum theory is the consequence of an idealization: that measurements are instantaneous. Canonical quantum theory can be given a covariant form by dropping this idealization. States prepared by non-instantaneous measurements are described by "spacetime smeared states". The theory can be formulated in terms of these states, without making any reference to a special time variable. The quantum dynamics is expressed in terms of the propagator, an object covariantly defined on the extended configuration space.
\end{abstract}

\section{INTRODUCTION}

In this paper, we discuss a covariant formulation of canonical quantum mechanics. This formulation is based on the propagator and on a representation of quantum states which we will call "spacetime-smeared quantum states". We think that this formalism can play a role in several problems, such as for instance: the interpretation of spinfoam quantum gravity, the interpretation of quantum cosmological models, certain interpretational issues in the quantum mechanics of a single relativistic particle and the problem of the computation of the time of arrival in quantum mechanics. These problems are all connected, and refer to the role that time plays in the formalism of quantum theory. Ideas closely related to the ones presented here can be found in the work of Jim Hartle [1.2] and Don Marolf [3,4]. But also in Bryce DeWitt [5], Chris Isham [6], John Klauder [7], Jonathan Halliwell [8], Rodolfo Gambini and Rafael Porto [9] and certainly others.

Our main tool is the spacetime-smeared representation of quantum states, which we define in Section [1]. This representation is the natural one for states prepared in measurements which are not instantaneous [2] and it is spacetime symmetric. We discuss in detail an example of such a measurement. We then construct a general formulation of quantum theory based on these states, in Section III. In Section IV, we apply this formulation to several concrete situations in order to test its viability and to point out its advantages. This formulation clarifies some issues in the formulation of the quantum theory of a single relativistic particle, and in relation to the time of arrival problem, and it helps us to give a consistent interpretation to quantum cosmological models. In Section $\mathrm{V}$, we discuss various conceptual issues raised by this formulation and in Section VI we briefly summarize.

One of the motivations for the present work is to help define the interpretation -that is, the relation between formalism and observation - of the formalisms for quantum gravity developed by the authors and others (see [10] and references therein) in which the central object that is computed is the propagator (or "projector on physical states") $P$. This work develops ideas on generally covariant quantum theory previously published by one of us (CR); however, the tools presented here allow a considerable simplification.

Finally, a note on notation: we use capital roman letters $(\mathrm{X}, \mathrm{T})$ for the space and time coordinates, while we use lower case italic letters $x, y, \ldots$ for spacetime points, and later on, for points in the extended configuration space. Thus for a particle in two dimensions $x=(\mathrm{X}, \mathrm{T})$.

\section{SPACETIME SMEARED QUANTUM STATES}

Consider a free, non-relativistic particle in one space dimension. Let $\psi(\mathrm{X}, \mathrm{T})$ be its Schrödinger wave function, namely a solution of the free Schrödinger equation 


$$
\imath \hbar \frac{\partial}{\partial \mathrm{T}} \psi(\mathrm{X}, \mathrm{T})=-\frac{\hbar^{2}}{2 m} \frac{\partial^{2}}{\partial \mathrm{X}^{2}} \psi(\mathrm{X}, \mathrm{T}) .
$$

The Hilbert space of the quantum theory is the space of normalizable solutions to the Schrödinger equation. It can be represented by the space $L_{2}[R]$ of square integrable functions on space alone. The wavefunction $\psi(X, T)$ is represented by the square integrable function $\Psi(\mathrm{X})=\psi(\mathrm{X}, 0)$ at a fixed time $\mathrm{T}=0$, and we will often denote the state by $|\Psi\rangle$. In this representation the scalar product is

$$
\left\langle\Psi \mid \Psi^{\prime}\right\rangle=\int d \mathrm{X} \overline{\Psi(\mathrm{X})} \Psi^{\prime}(\mathrm{X}) .
$$

The spacetime wavefunction $\psi$ can be reconstructed from $\Psi$ using the propagator. We denote the (generalized) eigenstates of the position operator $\mathrm{X}$ by $|\mathrm{X}\rangle$ and the generalized eigenstates of the unitarily evolving Heisenberg position operator $X(\mathrm{~T})$ as $|\mathrm{X}, \mathrm{T}\rangle$ (so that $|\mathrm{X}\rangle=|\mathrm{X}, 0\rangle$ ). Thus $\Psi(\mathrm{X})=\langle\mathrm{X} \mid \Psi\rangle$ and $\psi(\mathrm{X}, \mathrm{T})=\langle\mathrm{X}, \mathrm{T} \mid \Psi\rangle$. The propagator of the Schrödinger equation is

$$
\begin{aligned}
W\left(\mathrm{X}, T ; \mathrm{X}^{\prime}, \mathrm{T}^{\prime}\right) & =\left\langle\mathrm{X}, \mathrm{T} \mid \mathrm{X}^{\prime}, \mathrm{T}^{\prime}\right\rangle \\
& =\int \frac{d p}{2 \pi \hbar} d E e^{i / \hbar\left[p\left(\mathrm{X}-\mathrm{X}^{\prime}\right)-E\left(\mathrm{~T}-\mathrm{T}^{\prime}\right)\right]} \delta\left(E-p^{2} / 2 m\right) \\
& =\int \frac{d p}{2 \pi \hbar} e^{i / \hbar\left[p\left(\mathrm{X}-\mathrm{X}^{\prime}\right)-p^{2} / 2 m\left(\mathrm{~T}-\mathrm{T}^{\prime}\right)\right]} \\
& =\left(\frac{2 \pi m}{i \hbar\left(\mathrm{T}-\mathrm{T}^{\prime}\right)}\right)^{\frac{1}{2}} \exp \left\{-\frac{m\left(\mathrm{X}-\mathrm{X}^{\prime}\right)^{2}}{2 i \hbar\left(\mathrm{T}-\mathrm{T}^{\prime}\right)}\right\} .
\end{aligned}
$$

When viewed as a function of $X$ and $T$, with $X^{\prime}$ and $T^{\prime}$ held fixed, this is a solution of the Schrödinger equation which at time $\mathrm{T}=\mathrm{T}^{\prime}$ is given by a delta distribution in $\mathrm{X}-\mathrm{X}^{\prime}$. Each function $\Psi(\mathrm{X})$ determines a solution of the Schrödinger equation by

$$
\psi(\mathrm{X}, \mathrm{T})=\int d \mathrm{X}^{\prime} W\left(\mathrm{X}, \mathrm{T} ; \mathrm{X}^{\prime}, 0\right) \Psi\left(\mathrm{X}^{\prime}\right)
$$

Thus the wavefunctions allowed by the Schrödinger equation can be characterized by the functions $\Psi(X)$ of space only.

Now we shall consider another representation of quantum states. Consider the wavefunction given by

$$
\psi_{f}(\mathrm{X}, \mathrm{T}) \equiv \int d \mathrm{X}^{\prime} d \mathrm{~T}^{\prime} W\left(\mathrm{X}, \mathrm{T} ; \mathrm{X}^{\prime}, \mathrm{T}^{\prime}\right) f\left(\mathrm{X}^{\prime}, \mathrm{T}^{\prime}\right) .
$$

where $f(\mathrm{X}, \mathrm{T})$ is a smooth function on spacetime. The wave function $\psi_{f}(\mathrm{X}, \mathrm{T})$ is a solution of the Schrödinger equation as well. In the standard "instantaneous" representation discussed above, the wavefunction is represented by the function of space obtained by restricting $\psi_{f}(\mathrm{X}, \mathrm{T})$ to $\mathrm{T}=0$. If the restriction is square integrable the wavefunction is a normalizable state. We use the notation $|f\rangle$ for this state. That is

$$
|f\rangle=\int d \mathrm{X} d \mathrm{~T} f(\mathrm{X}, \mathrm{T})|\mathrm{X}, \mathrm{T}\rangle .
$$

Since the propagator satisfies the properties

$$
W\left(\mathrm{X}, \mathrm{T} ; \mathrm{X}^{\prime}, \mathrm{T}^{\prime}\right)^{*}=W\left(\mathrm{X}^{\prime}, \mathrm{T}^{\prime} ; \mathrm{X}, \mathrm{T}\right)
$$

and

$$
W\left(\mathrm{X}, \mathrm{T} ; \mathrm{X}^{\prime}, \mathrm{T}^{\prime}\right)=\int d \mathrm{X}^{\prime \prime} W\left(\mathrm{X}, \mathrm{T} ; \mathrm{X}^{\prime \prime}, \mathrm{T}^{\prime \prime}\right) W\left(\mathrm{X}^{\prime \prime}, \mathrm{T}^{\prime \prime} ; \mathrm{X}^{\prime}, \mathrm{T}^{\prime}\right),
$$

the scalar product between two such states is

$$
\left\langle f \mid f^{\prime}\right\rangle=\int d \mathrm{X} d \mathrm{~T} \int d \mathrm{X}^{\prime} d \mathrm{~T}^{\prime} \overline{f(\mathrm{X}, \mathrm{T})} W\left(\mathrm{X}, \mathrm{T} ; \mathrm{X}^{\prime}, \mathrm{T}^{\prime}\right) f^{\prime}\left(\mathrm{X}^{\prime}, \mathrm{T}^{\prime}\right) .
$$

It is easy to see that the spacetime smeared states $|f\rangle$ are dense in the Hilbert space of the theory. 
We shall call the normalizable state $|f\rangle$ labeled by the spacetime function $f(\mathrm{X}, \mathrm{T})$ a "spacetime-smeared quantum state", or simply a "spacetime state". It is important to notice that this denomination refers simply to the representation that the state is given. It is a perfectly ordinary normalizable quantum state in the ordinary Hilbert state of the theory.

It is clear that the spacetime-smeared representation is highly redundant - many different spacetime functions $f$ give rise to the same state - while the instantaneous representation is unique. This situation is a bit remeniscent of that found in electromagnetism, where the gauge freedom can be essentially eliminated in the Coulomb gauge, but at the cost of making the formalism non-covariant.

A particular class of spacetime states plays an important role in what follows. These are the spacetime states associated to small spacetime regions $\mathcal{R}$. Here "small" means smaller than any spatial or temporal scale involved in the problem being studied. We define the state $|\mathcal{R}\rangle$ associated to a spacetime region $\mathcal{R}$ as the normalized spacetime state defined by the characteristic function of $\mathcal{R}$, that is

$$
|\mathcal{R}\rangle=C_{\mathcal{R}} \int_{\mathcal{R}} d \mathrm{X} d \mathrm{~T} \quad|\mathrm{X}, \mathrm{T}\rangle
$$

The normalization factor is easily computed as

$$
C_{\mathcal{R}}=\left(\int_{\mathcal{R}} d \mathrm{X} d \mathrm{~T} \int_{\mathcal{R}} d \mathrm{X}^{\prime} d \mathrm{~T}^{\prime} W\left(\mathrm{X}, \mathrm{T} ; \mathrm{X}^{\prime}, \mathrm{T}^{\prime}\right)\right)^{-\frac{1}{2}}
$$

In the rest of this section we study the physical interpretation of the states $|f\rangle$ and the states $|\mathcal{R}\rangle$. In the rest of the paper we will use this spacetime-smeared representation and the propagator to construct a covariant formulation of canonical quantum theory.

Our central claim is (i) that the spacetime-smeared states $|f\rangle$ are natural objects, once one drops the unrealistic idealization that measurements are instantaneous, and (ii) that they make a spacetime symmetric formulation of quantum theory possible.

\section{A. Real measurements}

Roughly speaking, if we measure the position of the particle at time $\mathrm{T}=0$, and we find the particle in $\mathrm{X}=0$, we can then assume that the particle is in the state $|\mathrm{X}\rangle$. However, as is well known, no real measuring device can resolve a particle's position with infinite precision. Every real measuring device has a finite resolution $a$. We can represent a particle that at $\mathrm{T}=0$ has been detected in $\mathrm{X}=0$ by an apparatus with spatial resolution $a$ by a wave packet spread over a finite region of size $a$. Its state will have the form

$$
|\Psi\rangle=\int d \mathrm{X} f(\mathrm{X})|\mathrm{X}\rangle
$$

where $f(\mathrm{X})$ is, say, a function with support in the interval $[\mathrm{X}-a, \mathrm{X}+a]$, or, perhaps, a Gaussian smearing function

$$
f(\mathrm{X})=e^{-\frac{\mathrm{X}^{2}}{2 a}}
$$

What about time? In the usual textbook description, the measurement is assumed to be instantaneous. We observe, instead, that a real measuring device interacts with the system being measured for a finite interval of time. A real measurement never refers to a single sharply defined time [2]. Thus, in the case of a position measurement neither the position nor the time at which the particle is seen is resolved with infinite precision. Let's say that the measuring device resolves the time with precision $\epsilon$. We would like to claim that a particle detected in $\mathrm{X}=0$ at $\mathrm{T}=0$ with an experimental device having space resolution of order $a$ and time resolution of order $\epsilon$ can be described by a wave packet with the form

$$
|f\rangle=\int d \mathrm{X} d \mathrm{~T} f(\mathrm{X}, \mathrm{T})|\mathrm{X}, \mathrm{T}\rangle,
$$

where $f(\mathrm{X}, \mathrm{T})$ is a function concentrated in the region $[-a,+a] \times[-\epsilon,+\epsilon]$, such as for instance, the characteristic function of the region; or, say, by a Gaussian smearing function

$$
f(\mathrm{X}, \mathrm{T})=e^{-\frac{\mathrm{X}^{2}}{2 a}-\frac{\mathrm{T}^{2}}{2 \epsilon}} .
$$


Notice that in equation (14), the state is naturally represented as a spacetime-smeared state, as defined in the previous section.

In order to clarify this point, we now describe a simple model of a measurement procedure. For related constructions, see also [4]. This measurement procedure we describe is realistic in the sense that the physical interaction responsible for the measurement is not idealized away. We want to measure the position of the particle at a certain time. That is, we want to check whether the particle is present at a certain point $\mathrm{X}=0$ at a certain time $\mathrm{T}=0$. We thus set up a physical apparatus that interacts with the particle. This apparatus will have a pointer that tells us whether or not the particle has been detected. We now exploit the freedom in choosing the boundary between the quantum system under observation and the measuring apparatus: we treat the particle and particle detector as the system, and consider that the Copenhagen "measurement" is realized when the position of the pointer is observed. (In the measurement of the pointer, the time duration of the measurement is not an issue, because the pointer is static after the interaction with particle is complete.) This trick allows us to understand which precise aspect of the particle state is probed by an apparatus measuring the localization of the particle.

Let us consider for simplicity a pointer which has two possible states. A state $|0\rangle$, which corresponds to no detection, and a state $|1\rangle$, which corresponds to detection. We then represent the state space of the coupled particle-detector system by the Hilbert space $H_{P D}=H \otimes C^{2}$, where $H$ is the Hilbert space of the particle and $C^{2}$ is the state space of a two-state system. We write a state of the combined system as

$$
\Psi_{0}(\mathrm{X}) \otimes|0\rangle+\Psi_{1}(\mathrm{X}) \otimes|1\rangle .
$$

The free hamiltonian of the particle is $P^{2} / 2 m$, and we take the free hamiltonian of the detector to be zero. We need an interaction hamiltonian $H_{\text {int }}$, representing the interaction that gives rise to the measurement. $H_{\text {int }}$ must have the following properties. First, it must cause the transition $|0\rangle \rightarrow|1\rangle$. Second, the particle should interact only at or around the spacetime position $\mathrm{X}=0, \mathrm{~T}=0$. Thus the interaction hamiltonian must be time dependent, and vanish for late and early times. We have to concentrate the interaction around $\mathrm{T}=0$. However, we cannot have a perfectly instantaneous interaction because this would require infinite force. We must therefore assume that the interaction is non vanishing for a finite period of time. Putting these requirements together, and requiring also that the hamiltonian is self-adjoint, we arrive at an interaction hamiltonian of the form

$$
H_{\text {int }}=\alpha V(\mathrm{X}, \mathrm{T})(|1\rangle\langle 0|+| 0\rangle\langle 1|)
$$

where $\alpha V(\mathrm{X}, \mathrm{T})$ is the potential acting on the particle in the interaction (with $\alpha$ a coupling constant). The potential $V(\mathrm{X}, \mathrm{T})$ is concentrated in a finite spacetime region $\mathcal{R}$, which we take to be concentrated around $\mathrm{X}=0$ and $\mathrm{T}=0$.

The Schrödinger equation for the spacetime wavefunctions of the particle states $\Psi_{0}$ and $\Psi_{1}$ reads

$$
\begin{aligned}
& \imath \hbar \frac{\partial}{\partial \mathrm{T}} \psi_{0}(\mathrm{X}, \mathrm{T})=-\frac{\hbar^{2}}{2 m} \frac{\partial^{2}}{\partial \mathrm{X}^{2}} \psi_{0}(\mathrm{X}, \mathrm{T})+\alpha V(\mathrm{X}, \mathrm{T}) \psi_{1}(\mathrm{X}, \mathrm{T}) \\
& \imath \hbar \frac{\partial}{\partial \mathrm{T}} \psi_{1}(\mathrm{X}, \mathrm{T})=-\frac{\hbar^{2}}{2 m} \frac{\partial^{2}}{\partial \mathrm{X}^{2}} \psi_{1}(\mathrm{X}, \mathrm{T})+\alpha V(\mathrm{X}, \mathrm{T}) \psi_{0}(\mathrm{X}, \mathrm{T}) .
\end{aligned}
$$

Now assume that at some early time $\mathrm{T}_{i n}<<0$ the particle is in some initial state $\psi\left(\mathrm{X}, \mathrm{T}_{i n}\right)$ and the pointer is in the state $|0\rangle$. What is the state of the system at a later time $\mathrm{T}_{\text {fin }}>>0$ ? It is straightforward to integrate the evolution equations to first order in $\alpha$. One obtains

$$
\begin{aligned}
\psi_{0}(\mathrm{X}, \mathrm{T}) & =\int d \mathrm{X}^{\prime} W\left(\mathrm{X}, \mathrm{T} ; \mathrm{X}^{\prime}, \mathrm{T}_{i n}\right) \psi_{0}\left(\mathrm{X}^{\prime}, \mathrm{T}_{i n}\right) . \\
\psi_{1}\left(\mathrm{X}, \mathrm{T}_{f i n}\right) & =\frac{\alpha}{i \hbar} \int_{\mathcal{R}} d \mathrm{X}^{\prime} d \mathrm{~T}^{\prime} W\left(\mathrm{X}, \mathrm{T}_{f i n} ; \mathrm{X}^{\prime}, \mathrm{T}^{\prime}\right) V\left(\mathrm{X}^{\prime}, \mathrm{T}^{\prime}\right) \psi_{0}\left(\mathrm{X}^{\prime}, \mathrm{T}^{\prime}\right) .
\end{aligned}
$$

If the pointer is observed in the state $|1\rangle$ after the interaction, the state of the system collapses to $\Psi_{1} \otimes|1\rangle$. After the measurement, the state of the particle is thus described by the wave function (21). But notice that this has precisely the form (14) of a spacetime smeared state, where the spacetime smearing function has support in $\mathcal{R}$ :

$$
f(\mathrm{X}, \mathrm{T})=V(\mathrm{X}, \mathrm{T}) \psi_{0}(\mathrm{X}, \mathrm{T}) .
$$

The result to all orders in perturbation theory is more complicated, but it is easy to see that $\psi_{1}$ still has the form of a spacetime smeared state with $f$ supported in $\mathcal{R}$. Thus we can conclude that if we prepare a state concentrated around $\mathrm{X}=0, \mathrm{~T}=0$ by means of a physical measurement procedure like the one described, we necessarily obtain a 
spacetime-smeared state defined by a function $f(\mathrm{X}, \mathrm{T})$ with support in a finite region $\mathcal{R}$ around $\mathrm{X}=0, \mathrm{~T}=0$. The size of $\mathcal{R}$ is determined by the accuracy of the measuring apparatus in resolving distances and time intervals.

Consider now an ideal case in which the region $\mathcal{R}$ is much smaller than the size over which the wave function $\psi(\mathrm{X}, \mathrm{T})$ varies and the potential $V(\mathrm{X}, \mathrm{T})$ is constant over $\mathcal{R}$. In this case, if the pointer is observed in the state $|1\rangle$ after the interaction, the state of the particle collapses precisely to the state $|\mathcal{R}\rangle$, defined in (10 11). The dependence of the final state on the initial wave function, on $\alpha$ and on the potential, is completely cancelled by the normalization of the state.

The model of detector that we have described is only an example, but we think that the conclusion that no position detector can have infinite time resolution is true in general. Textbook model detectors have elements of idealization that hide the finite time resolution. We shall not attempt a general analysis. We close this subsection, however, by showing that the measurement time is finite also for the quintessential position detector: a hole in a wall. Consider a physical particle in three spacial dimensions $X, Y$ and $Z$. Suppose we want to prepare a state in which the coordinates $Y$ and $Z$ are concentrated in a finite (two-dimensional) interval $I$. Then we can take a wall in the $(Y, Z)$ plane, having a hole of dimension $I$, and have the particle go through. If the particle passes accross the hole, then its $Y$ and $Z$ coordinates are in $I$. This is the simplest position measurement discussed in textbooks. At which time such a measurement happens? In the usual textbook discussion, one takes a state described by a plane wave moving in the $X$ direction, namely normal to the wall. This can be interpreted as describing a steady flux of particles, and any time consideration is thus avoided. But what if we have a single particle? Then the time of the measurement is clearly the time $T$ at which the particle reaches the wall and crosses (or fails to cross) the hole. But the true state of the particle cannot be infinitely concentrated in the $X$ direction. The particle will be described by a wave packet that has a finite spread $\Delta X$ in the $X$ direction and a spread $\Delta V$ in the velocity in the $Y$ direction. Accordingly, the wave packet will cross the hole during a time period of the order $\epsilon \sim \Delta X / \Delta V$. The source of the component of the wave function emerging on the other side of the hole is thus concentrated in the spacetime region $\mathcal{R}=(T \pm \epsilon) \times I$, and not in the region $I$ at a fixed time $T$. Again, we conclude that the natural description of the state emerging from the hole is in terms of a spacetime-smeared state, not as an eigenstate of a position projection operator at fixed time.

\section{B. The states $|\mathcal{R}\rangle$ and ideal spacetime measurements}

The interpretation of Schrödinger quantum mechanics is based on the postulate that $|\psi(\mathrm{X}, \mathrm{T})|^{2}$ is the spatial probability density of finding the particle at X, at time T. Equivalently, one can consider a small (smaller than any spatial scale in the problem) spatial interval $I=[x, x+\Delta x]$ and the normalized state $|I\rangle$ which is constant over $I$. This state has two properties, first, it represents a possible state prepared by a measurement of spatial position in $I$. Second, the probability that an ideal detector (a detector with efficiency 1 ) find the particle in $I$ is $|\langle I \mid \Psi\rangle|^{2}$.

We are searching for a spacetime version of this interpretation. Do the states $|\mathcal{R}\rangle$ associated to spacetime regions have analogous properties? In the previous subsection we have seen that a certain measurement prepares the state

\footnotetext{
${ }^{1}$ The apparatus we have described is effective for preparing a quantum state concentrated around the origin, but performs poorly as a detector of whether a particle is or not around the origin. This is because there is always a finite probability for the pointer to "forget", namely to jump back from $|1\rangle$ to $|0\rangle$, after the first detection. This does not happen to first order in $\alpha$, but it is easy to see that it happens to second order. We can minimize the probability of "forgetting" by taking $\alpha$ small, but this gives us a detector which works correctly, but has low efficiency. Real detectors, however, are dissipative. For instance, in the silver nitrate crystals of photographic film, the microscopic signal that is being detected triggers the fall of the detector to a lower energy, with the energy liberated being absorbed by the environment, raising it's entropy. There is one, or a small number, of states corresponding to no detection, but a large number of states corresponding to detection. It follows that once the detector (and environment) has interacted with the microsystem it cannot find it's way back to it's initial state in a second interaction, even though this is energetically possible, for reasons of statistics. A simple way of taking this fact into account in the model is to replace (17) with the interaction hamiltonian

$$
H_{\text {int }}=\alpha V(\mathrm{X}, \mathrm{T})|1\rangle\langle 0|
$$

which is not self-adjoint. With this hamiltonian, the solution (21) is indeed exact not just to first order, but to all orders in $\alpha$ : once the detector has detected the particle, it does not forget it. We are thus not forced to take $\alpha$ small, and we can use such a detector not only to prepare a quantum state concentrated around the origin, but also to efficiently check whether a particle is at the orgin or not.
} 
$|\mathcal{R}\rangle$. Can we also say that the probability $P$ that an ideal detector detects the particle in $\mathcal{R}$ is $|\langle\mathcal{R} \mid \Psi\rangle|^{2}$ ? We now show that the answer is yes.

The probability of detection of the particle is given by the norm of the state entangled with the "yes" position of the pointer, that is with $|1\rangle$. It is thus

$$
\begin{aligned}
P & =\int d \mathrm{X}\left|\psi_{1}\left(\mathrm{X}, \mathrm{T}_{f i n}\right)\right|^{2} \\
& =\frac{\alpha}{\hbar} \int d \mathrm{X} \int_{\mathcal{R}} d \mathrm{X}^{\prime} d \mathrm{~T}^{\prime} \overline{W\left(\mathrm{X}, \mathrm{T}_{f i n} ; \mathrm{X}^{\prime}, \mathrm{T}^{\prime}\right) \psi_{0}\left(\mathrm{X}^{\prime}, \mathrm{T}^{\prime}\right)} \frac{\alpha}{\hbar} \int_{\mathcal{R}} d \mathrm{X}^{\prime \prime} d \mathrm{~T}^{\prime \prime} W\left(\mathrm{X}, \mathrm{T}_{f i n} ; \mathrm{X}^{\prime \prime}, \mathrm{T}^{\prime \prime}\right) \psi_{0}\left(\mathrm{X}^{\prime \prime}, \mathrm{T}^{\prime \prime}\right) .
\end{aligned}
$$

Integrating in $d \mathrm{X}$ and using the properties of the propagator this gives

$$
P=\frac{\alpha^{2}}{\hbar^{2}} \int_{\mathcal{R}} d^{2} x \int_{\mathcal{R}} d^{2} y \overline{\psi_{0}(x)} W(x ; y) \psi_{0}(y),
$$

where we have switched to the spacetime notation $x=(\mathrm{X}, \mathrm{T})$.

If $\mathcal{R}$ is sufficiently small that the wavefunction $\psi_{0}$ of the incident particle is well approximated in $\mathcal{R}$ by its value $\psi_{0}(x)$ at a point $x=(X, T) \in \mathcal{R}$ then

$$
P=\left(\frac{\alpha}{\hbar C_{\mathcal{R}}}\right)^{2}\left|\psi_{0}(x)\right|^{2} .
$$

On the other hand the overlap of the particle's state with the characteristic state $|\mathcal{R}\rangle$ is (always in the small region limit)

$$
\langle\mathcal{R} \mid \Psi\rangle=C_{\mathcal{R}} \int_{\mathcal{R}} \psi_{0}(X, T) d X d T=C_{\mathcal{R}} V_{\mathcal{R}} \psi_{0}(x),
$$

where $V_{\mathcal{R}}$ is the spacetime volume of $\mathcal{R}$. Thus the the probability of detection is

$$
P=\gamma|\langle\mathcal{R} \mid \Psi\rangle|^{2},
$$

where

$$
\gamma=\left(\frac{\alpha}{\hbar V_{\mathcal{R}} C_{\mathcal{R}}^{2}}\right)^{2},
$$

which depends on the detector only, can be interpreted as the detector's efficiency.

If the particle is in the state $|\mathcal{R}\rangle$ the detector is triggered with efficiency $\gamma$, while if the particle is in a state orthogonal to $|\mathcal{R}\rangle$, the detector is certainly not triggered.

The detector realizes another characteristic of an ideal detector of the state $|\mathcal{R}\rangle$ : if it is triggered, and the particle was already in the state $|\mathcal{R}\rangle$, the detector leaves the particle in the state $|\mathcal{R}\rangle$.

Since we have used first order perturbation theory, the validity of our calculation requires that $\gamma \ll 1$, as can be seen by calculating the second order correction. The detector we have analysed has therefore low efficiency: it usually misses particles which would have triggered an ideal detector with efficiency 1 . Nevertheless the low efficiency detector can equally be used to test the predicted values of the probability $|\langle\mathcal{R} \mid \Psi\rangle|^{2}$, by simply comparing the predicted values with the observed detection frequencies multiplied by the calibration factor $1 / \gamma$. We can therefore define the notion of an ideal detector, whose efficiency is one, and whose probability dectection is $|\langle\mathcal{R} \mid \Psi\rangle|^{2}$.

We thus conclude that when $\mathcal{R}$ is sufficiently small the states $|\mathcal{R}\rangle$ are prepared and are detected by a particle detector in the spacetime region $\mathcal{R}$. The detection probabilities of the ideal detector are

$$
P_{\mathcal{R}}=|\langle\mathcal{R} \mid \Psi\rangle|^{2} .
$$

Equation (30) derives from the usual probabilistic interpretation of the wave function. In the next section we will show that the usual probabilistic interpretation can, in turn, be derived from (30). Therefore (30) is physically equivalent to the standard interpretation in terms of the probabities of detection in spatial (equal-time) regions. However, it does not refer to a preferred time coordinate. Equation (30) is a key result of this paper: we shall take it as the central ingredient of the interpretation of the covariant formulation of quantum theory.

The quantity $P_{\mathcal{R}}$ for small regions $\mathcal{R}$ provide a probabilistic interpretation of the modulus of the wave function. The probabilities for large $\mathcal{R}$ or $\mathcal{R}$ consisting of two small separated components depend on the relative phase of the 
wavefunction at different spacetime points, thus providing the probabilistic interpretation of the (relative) phase, as in the standard situation. As usual, if the $P_{\mathcal{R}}$ are measured for all $\mathcal{R}$ on (separate instances of) the same state they characterize the wavefunction completely (up to the overall phase).

Finally, the result that we have obtained can be expressed in the stardard operator language as follows. A (efficiency one) measurement of whether the particle is in the small spacetime region $\mathcal{R}$ is represented by the self-adjoint operator

$$
\Pi_{\mathcal{R}}=|\mathcal{R}><\mathcal{R}| .
$$

The corresponding classical observable has value 1 on all the solutions of the equation of motion that cross $\mathcal{R}$, and zero elsewhere.

\section{Relation with the spatial probability density}

We have seen above that starting from standard quantum theory one can derive the interpretation of $P_{\mathcal{R}}=|\langle\mathcal{R} \mid \Psi\rangle|^{2}$ as probability that the particle is detected in $\mathcal{R}$. Here, for completeness, we show how one go back from the probability in spacetime $P_{\mathcal{R}}$ to the standard interpretation of $|\psi|^{2}$ as a probability in space, and we discuss why spacetime probability densities cannot be defined.

The probability to detect the particle in a small region $\mathcal{R}$ is given by (30). Consider small rectangular spacetime regions $\mathcal{R}$ of spatial width $\Delta X$ and duration $\Delta T$ which satisfy the inequality $\Delta T \ll m \Delta X^{2} / \hbar$. This region can be thought as a rectangle with the timelike side much smaller than the spacial side. It is not hard to prove that for such a region, and up to higher order terms in the size of the region,

$$
C_{\mathcal{R}}^{-2}=\int_{\mathcal{R}} d^{2} x \int_{\mathcal{R}} d^{2} y W(x ; y)=\Delta X \Delta T^{2}
$$

(because $\int_{-\infty}^{\infty} d X W\left(X, T ; X^{\prime}, T^{\prime}\right)=1$ and $\mathcal{R}$ is suficiently spatially wide compared to it's duration that $X$ integrals give almost the same result as the integral over $(-\infty, \infty))$. Consequently, for these regions we have

$$
P_{\mathcal{R}}=|\langle\mathcal{R} \mid \Psi\rangle|^{2}=C_{\mathcal{R}}^{2}\left|\int_{\mathcal{R}} \psi(x) d^{2} x\right|^{2}=|\psi(x)|^{2} \frac{\Delta X^{2} \Delta T^{2}}{\Delta X \Delta T^{2}}=|\psi(x)|^{2} \Delta X
$$

(where $x$ is again a point in the small region $\mathcal{R}$ ). We have therefore two results. First, the detection probability does not depend on the duration of $\mathcal{R}$ for such regions. This is in fact the key reason for which the duration of a measurement can be safely neglected in the quantum theory of a particle: if this duration is sufficiently short, the probability to detected the particle is independent from such a duration. Second, the probability is proportional to the spatial extension of the interval. Therefore we can define a spatial probability density $\rho(x)$ by

$$
\rho(x)=\lim _{\Delta L \rightarrow 0} \frac{P_{\mathcal{R}}}{\Delta L},
$$

and we obtain that the probability density in space is

$$
\rho(x)=|\psi(x)|^{2} .
$$

Cover a spatial region $\Omega$ at time $T$ by many contigous but non overlapping small rectangular spacetime regions $\mathcal{R}_{n}$ of the type we have been considering. If we assemble the detectors of the corresponding characteristic states $\left|\mathcal{R}_{n}\right\rangle$, we can ask what is the probability that the particle will be detected by any of the detectors. Detection by different detectors are mutually exclusive alternatives since their associated characteristic states are orthogonal: $\int_{\mathcal{R}} d^{2} x \int_{\mathcal{R}^{\prime}} d^{2} y W(x ; y) \simeq 0$ when $\mathcal{R}$ and $\mathcal{R}^{\prime}$ are simultaneous, non-overlapping, and both have dimensions obeying $\Delta T \ll m \Delta X^{2} / \hbar$. Thus the probability is just the sum of the individual detection probabilities.

$$
P=\sum_{n} P_{\mathcal{R}_{n}} \simeq \sum_{n}\left(|\psi|^{2} \Delta X\right)_{n} \rightarrow \int_{\Omega}|\psi|^{2} d X
$$

where the last expression is exact in the limit of infinitesimal $\Delta X$, and is accurate as long as $\psi$ is approximately constant in each $\mathcal{R}_{n}$. What we have found is of course precisely the standard probability interpretation of the wavefunction. When applied to the non-relativistic quantum mechanics of a single particle using the propagator specific to this system, the covariant probability interpretation (30) yields the standard probability interpretation of the wavefunction of this system. 
It is important to notice that the fact that detection in disjoint spatial regions at equal time are mutually exclusive alternatives does not reflect a special role of time in the formalism but rather is a feature of the propagator of the non-relativistic particle.

Finally, can we define the probability density in spacetime to find the particle around a spacetime point $x$ ? The answer is no, for the following reason. In order to be able to define such a probability, the following limit should exist

$$
\tilde{\rho}(x)=\lim _{\mathcal{R} \rightarrow x} \frac{P_{\mathcal{R}}}{V_{\mathcal{R}}}
$$

where $V_{\mathcal{R}}$ is the volume of the region. Consider a region $\mathcal{R}$ of sides $\epsilon L$ and $\epsilon T$. A tedious integral shows that for small $\epsilon$

$$
C_{\mathcal{R}}^{-2} \simeq \sqrt{\frac{\hbar}{m}} L^{2} T^{3 / 2} \epsilon^{7 / 2}
$$

Thus

$$
P_{\mathcal{R}}=|\langle\mathcal{R} \mid \Psi\rangle|^{2}=C_{\mathcal{R}}^{2} V_{\mathcal{R}}^{2}|\psi(x)|^{2}=|\psi(x)|^{2}(\epsilon T)^{-1 / 2},
$$

so that the probability does not scale with the volume, and the limit (37) does not exist. Therefore there is no probability density in spacetime.

\section{Towards a covariant formulation of quantum theory}

The possibility of labeling quantum states by means of spacetime functions $f(\mathrm{X}, \mathrm{T})$, or more generally functions of the configuration space and time, opens the possibility of formulating ordinary quantum theory in a form in which the time variable plays a less peculiar role than in the conventional formulation. To prepare the ground for such a reformulation we begin here by reviewing the structures we have introduced in the context of the non-relativistic free particle in a more abstract mathematical manner, so that they can then be generalized.

First, let $\mathcal{H}$ be the linear space of the physical solutions $\psi(\mathrm{X}, \mathrm{T})$ of the Schrödinger equation. $\mathcal{H}$ is canonically isomorphic to the Hilbert space $\mathcal{H}_{0}$ of the states $\Psi(\mathrm{X})$ at fixed time $\mathrm{T}=0$ : the identification map $I: \mathcal{H} \rightarrow \mathcal{H} 0$ is given by the restriction $\Psi(\mathrm{X})=\psi(\mathrm{X}, 0)$ and its inverse $I^{-1}: \mathcal{H}_{0} \rightarrow \mathcal{H}$ is given by the Schrödinger evolution

$$
\psi(\mathrm{X}, \mathrm{T})=\int d \mathrm{X}^{\prime} W\left(\mathrm{X}, \mathrm{T} ; \mathrm{X}^{\prime}, 0\right) \Psi\left(\mathrm{X}^{\prime}\right)
$$

The identification map induces the physical Hilbert product on $\mathcal{H}$ by

$$
\left(\psi, \psi^{\prime}\right)_{\mathcal{H}} \equiv\left(I \psi, I \psi^{\prime}\right)_{\mathcal{H}_{0}}=\int d \mathrm{X} \overline{\psi(\mathrm{X}, 0)} \psi^{\prime}(\mathrm{X}, 0)
$$

Next, consider a space $\mathcal{E}$ formed by spacetime "test functions" $f(\mathrm{X}, \mathrm{T})$. For concreteness, we take these functions to be smooth and with compact support. More general functions such as rapid decrease functions, can be more convenient for some applications. A very important object is the linear map $P$ from the space of the test functions $\mathcal{E}$ to the Hilbert space $\mathcal{H}$, defined by

$$
\begin{aligned}
P: \mathcal{E} & \rightarrow \mathcal{H} \\
: f & \mapsto \mid f> \\
\psi_{f}(\mathrm{X}, \mathrm{T}) \equiv<\mathrm{X}, \mathrm{T} \mid f> & \equiv \int d \mathrm{X}^{\prime} d \mathrm{~T}^{\prime} W\left(\mathrm{X}, \mathrm{T} ; \mathrm{X}^{\prime}, \mathrm{T}^{\prime}\right) f\left(\mathrm{X}^{\prime}, \mathrm{T}^{\prime}\right) .
\end{aligned}
$$

(See equation (5).) This map is highly degenerate: it sends arbitrary functions in solutions of the Schrödinger equation. Its image is dense in $\mathcal{H}$. The scalar product can be pulled back to $\mathcal{E}$, giving

$$
\left\langle f \mid f^{\prime}\right\rangle=\int d \mathrm{X} d \mathrm{~T} \int d \mathrm{X}^{\prime} d \mathrm{~T}^{\prime} \overline{f(\mathrm{X}, \mathrm{T})} W\left(\mathrm{X}, \mathrm{T} ; \mathrm{X}^{\prime}, \mathrm{T}^{\prime}\right) f^{\prime}\left(\mathrm{X}^{\prime}, \mathrm{T}^{\prime}\right) .
$$

(See equation (9).) And therefore the Hilbert space of the theory $\mathcal{H}$ is nothing but the linear space $\mathcal{E}$ equipped by the bilinear form (43) (divised by the zero norm subspace and completed in norm). Therefore the propagator $W(x, y)$ contains the full information needed to reconstruct the Hilbert space of the theory from the linear space $\mathcal{E}$. 
Let $C$ be the Schrödinger operator defined on $\mathcal{E}$

$$
C=\imath \hbar \frac{\partial}{\partial \mathrm{T}}+\frac{\hbar^{2}}{2 m} \frac{\partial^{2}}{\partial \mathrm{X}^{2}}
$$

Can $W(x, y)$ be recovered directly from the operator $C$ (without passing via the $|\mathrm{X}, \mathrm{T}\rangle$ states as we did in section II)? The answer is positive, and there is a number of general techniques to derive $W(x, y)$ directly from $C$. Here we describe a general technique denoted group averaging, essentially following Marolf's ideas [3]. We refer the reader to [3] and references therein for more details and a for more complete mathematical treatement of the technique. The operator $C$ defines on $\mathcal{E}$ the bilinear form

$$
\left(f^{\prime}, f\right)_{C} \equiv \int_{-\infty}^{\infty} d \tau \int d \mathrm{X} d \mathrm{~T} \overline{f^{\prime}(\mathrm{X}, \mathrm{T})}\left[e^{i \tau C} f\right](\mathrm{X}, \mathrm{T}) .
$$

This can be easily computed by Fourier transforming, obtaining

$$
\begin{aligned}
\left(f^{\prime}, f\right)_{C} & =\int_{-\infty}^{\infty} d \tau \int d \mathrm{X} d \mathrm{~T} d \mathrm{X}^{\prime} d \mathrm{~T}^{\prime} d p d E e^{-i p \mathrm{X}^{\prime}} e^{-i E \mathrm{~T}^{\prime}} \tilde{f}(-p,-E) e^{i \tau\left(E+p^{2} / 2 m\right)} e^{i p \mathrm{X}} e^{i E \mathrm{~T}} \tilde{f}(p, E) \\
& =\int d p d E \tilde{f}(-p,-E) \delta\left(E+p^{2} / 2 m\right) \tilde{f}(p, E) \\
& =\int d \mathrm{X}^{\prime} d \mathrm{~T}^{\prime} d \mathrm{X} d \mathrm{~T} \overline{f^{\prime}\left(\mathrm{X}^{\prime}, \mathrm{T}^{\prime}\right)} W\left(\mathrm{X}^{\prime}, \mathrm{T}^{\prime}, \mathrm{X}, \mathrm{T}\right) f(\mathrm{X}, \mathrm{T}) .
\end{aligned}
$$

Therefore the propagator $W(x, y)$ is nothing by the kernel of the bilinear form $(,)_{C}$ defined in 44). In turn, this bilinear form is precisely the scalar product. Once more, we have:

$$
\begin{aligned}
\left(P f^{\prime}, P f\right)_{\mathcal{H}}= & \int d \mathrm{X} \overline{\psi_{f^{\prime}}(\mathrm{X}, 0)} \psi_{f}(\mathrm{X}, 0) \\
= & \int d \mathrm{X} d \mathrm{X}^{\prime} d \mathrm{~T}^{\prime} d \mathrm{X}^{\prime \prime} d \mathrm{~T}^{\prime \prime} \\
& \quad \times \overline{W\left(\mathrm{X}, 0 ; \mathrm{X}^{\prime} \mathrm{T}^{\prime}\right) f^{\prime}\left(\mathrm{X}^{\prime}, \mathrm{T}^{\prime}\right)} W\left(\mathrm{X}, 0 ; \mathrm{X}^{\prime} \mathrm{T}^{\prime}\right) f^{\prime}\left(\mathrm{X}^{\prime \prime}, \mathrm{T}^{\prime \prime}\right) \\
= & \int d \mathrm{X}^{\prime} d \mathrm{~T}^{\prime} d \mathrm{X}^{\prime \prime} d \mathrm{~T}^{\prime \prime} \overline{f^{\prime}\left(\mathrm{X}^{\prime}, \mathrm{T}^{\prime}\right)} W\left(\mathrm{X}^{\prime}, \mathrm{T}^{\prime} ; \mathrm{X}^{\prime} \mathrm{T}^{\prime}\right) f^{\prime}\left(\mathrm{X}^{\prime \prime}, \mathrm{T}^{\prime \prime}\right) \\
= & \left(f^{\prime}, f\right)_{C} .
\end{aligned}
$$

Therefore $P$ maps isometrically the linear space $\mathcal{E}$ equipped with the bilinear form $(,)_{C}$ into the Hilbert space $\mathcal{H}$ of the theory. As $P \mathcal{E}$ is dense in $\mathcal{H}$, it follows that $\mathcal{H}$ is entirely determined by $\mathcal{E}$ and $(,)_{C}$.

This is a remarkable conclusion, because one often finds in the literature the statement that in order to define the scalar product on the space of the solutions of the Schrödinger equation one has first to identify $\mathrm{T}$ as the time variable. Contrary to this statement, we see here that, at least for this simple case, the Hilbert space of the theory including its scalar product structure is entirely determined over a space of functions on spacetime by the Schrödinger operator $C$, without having to single out the variable $\mathrm{T}$ as "special".

\section{GENERAL COVARIANT QUANTUM THEORY}

Let us now leave the simple case of a free particle, and consider the general situation. Consider a classical dynamical system. The kinematics of the system is defined by an (extended) configuration space $\mathcal{M}$. We call $x$ the points in $\mathcal{M}$, and we assume that a measure $d x$ is fixed. The dynamics of the system is defined by a (single for simplicity) constraint $C=0$. Here $C$ is a function on $\Gamma$, where $\Gamma=T^{*} \mathcal{M}$ is the (extended) phase space, namely the cotangent space of $\mathcal{M}$. The couple $(\mathcal{M}, C)$ completely defines the system. We call here this formulation of classical dynamics "covariant". Other denominations in the literature are presymplectic, parametrized, extended ...

It is well known that a conventional hamiltonian system can be cast in this form. A conventional hamiltonian system is formulated in terms of a configuration space $\mathcal{M}_{p h}$ and a hamiltonian $H$ which is a function on the phase space $\Gamma_{P h}=T^{*} \mathcal{M}_{p h}$. The hamiltonian generates evolution in an external time variable $\mathrm{T}$. To reformulate this system in covariant form, one promotes $\mathrm{T}$ to a configuration space variable: the extended configuration space includes the conventional configuration space plus the time $T$. That is, one poses $\mathcal{M}=\mathcal{M}_{p h} \times \mathbb{R}$, where the coordinate of $\mathbb{R}$ is 
identified with $\mathrm{T}$. Also, one poses $C=p_{T}+H$, where $p_{T}$ is the variable conjugate to $\mathrm{T}$ (which physically turns out to be minus the energy).

A (well known) crucial observation is that most interesting physical systems, and in particular all gravitational systems, such as full general relativity with or without matter, cosmological models ... are not given in terms of a hamiltonian: they are given directly in the covariant formulation. Therefore not only the covariant formulation of mechanics appears to be more general than the hamiltonian one, but such a wider generality is required for the theories that better describe our world.

One can try to "deparametrize" these theories by picking one of the configuration space space variables and identifying it as the time variable. It is sometimes claimed that such a deparametrization is necessary in order to understand the quantum properties of the these systems. But such a deparametrization adds an element of arbitrariness which is certainly not part of the classical dynamics. Since the classical dynamics of these systems does not select any preferred independent "time" variable $\mathrm{T}$, we think that their quantum mechanics should not select a preferred time variable either. To understand their quantum dynamics, we must therefore have a formulation of quantum theory in which time plays no special role. This is the motivation for the definition of covariant quantum theory that we give in this section.

We want thus to quantize the system $(\mathcal{M}, C)$. We begin with a space $\mathcal{E}$ of test functions $f(x)$ over $\mathcal{M}$. (We use now latin letters $x, y, \ldots$ for points in the extended configuration space.) The quantum dynamics is then determined by a propagator $W(x, y)$ on $\mathcal{M} \times \mathcal{M}$.

Here we are more interested in the interpretation of the theory once the propagator is given, than in the actual construction of the propagator. Let us nevertheless say something on the derivation of $W(x, y)$ itself. There is a number of ways of constructing this object starting from the classical theory. For instance, $W(x, y)$ may be defined as a sum over classical histories (11. In the case of nonperturbative quantum gravity, $W(x, y)$ may be defined by means of an auxiliary quantum field over a group [10]. In a canonical quantization, assume that an operator $C=C\left(x,-i \hbar \frac{\partial}{\partial x}\right)$ whose classical limit is the constraint $C$ is given (that is, assume a given operator ordering has been chosen). One can then follow closely Marolf's construction [3] mentioned in the previous section. That is, define a bilinear form on $\mathcal{E}$

$$
\left(f^{\prime}, f\right)_{C} \equiv \int_{-\infty}^{\infty} d \tau \int d x \overline{f^{\prime}(x)}\left[e^{i \tau C} f\right](x) .
$$

as in equation (45). See [3]. The kernel of this bilinear form defines $W(x, y)$ :

$$
\left(f, f^{\prime}\right)_{C}=\int d x d x^{\prime} \overline{f(x)} W\left(x, x^{\prime}\right) f\left(x^{\prime}\right) .
$$

Once $W(x, y)$ is determined, the rest of the formalism and the interpretation of the quantum theory follow. The Hilbert space of the theory is defined as the space $\mathcal{H}$ obtained by equipping $\mathcal{E}$ with the bilinear form (49), quotienting by the kernel of the bilinear form and completing in norm. The physical states can therefore be labelled by the functions on $\mathcal{M}$ as $|f\rangle$. The (highly degenerate) map

$$
\begin{aligned}
P & : \mathcal{E} \rightarrow \mathcal{H} \\
& : f \mapsto \mid f>
\end{aligned}
$$

is often improperly called the "projector".

Each state $|f\rangle$ determines a solution of the quantum constraint equation via

$$
\psi_{f}(\mathrm{X}, \mathrm{T}) \equiv \int d \mathrm{X}^{\prime} d \mathrm{~T}^{\prime} W\left(\mathrm{X}, \mathrm{T} ; \mathrm{X}^{\prime}, \mathrm{T}^{\prime}\right) f\left(\mathrm{X}^{\prime}, \mathrm{T}^{\prime}\right) .
$$

The scalar product on the space of these solutions, is well defined by $\left\langle f \mid f^{\prime}\right\rangle=\left(f, f^{\prime}\right)_{C}$, namely by (49). But in general it may not be easilly written directly in terms of the solutions $\psi_{f}(\mathrm{X}, \mathrm{T})$ themselves.

We must then give the theory an interpretation. Associate a (normalized) state $|\mathcal{R}\rangle$ to each finite region $\mathcal{R}$ in $\mathcal{M}$. The state $|\mathcal{R}\rangle$ is defined as

$$
|\mathcal{R}\rangle=C_{\mathcal{R}}\left|f_{\mathcal{R}}\right\rangle,
$$

where $f_{\mathcal{R}}$ is the characteristic function of the region $\mathcal{R}$ and $C_{\mathcal{R}}=\left|\left\langle f_{\mathcal{R}} \mid f_{\mathcal{R}}\right\rangle\right|^{-1 / 2}$ is the normalization factor. We define the interpretation of the theory by postulating that if $\mathcal{R}$ is sufficiently small (namely in the limit in which $\mathcal{R}$ is still finite, but smaller than any other physically relevant dimension involved in the problem), the probability amplitude to detect a system (in the state $|\Psi\rangle$ ) in the region $\mathcal{R}$ is given by 


$$
P_{\mathcal{R}}=|\langle\mathcal{R} \mid \Psi\rangle|^{2}
$$

In turn, $|\mathcal{R}\rangle$ represents the state of the system after a measurement that has detected the system in the region $\mathcal{R}$.

In particular, the quantity

$$
A_{\mathcal{R}, \mathcal{R}^{\prime}}=\left\langle\mathcal{R} \mid \mathcal{R}^{\prime}\right\rangle
$$

is the probability amplitude to detect the system in the (small) region $\mathcal{R}$ of the extended configuration space, if the system was previously detected in the (small) region $\mathcal{R}^{\prime}$. This amplitude can be written explicitely in terms of the propagator as

$$
A_{\mathcal{R}, \mathcal{R}^{\prime}}=C_{\mathcal{R}} C_{\mathcal{R}^{\prime}} \int_{\mathcal{R}} d x \int_{\mathcal{R}^{\prime}} d y W(x ; y)=\frac{\int_{\mathcal{R}} d x \int_{\mathcal{R}^{\prime}} d y W(x ; y)}{\sqrt{\int_{\mathcal{R}} d x \int_{\mathcal{R}} d y W(x ; y)} \sqrt{\int_{\mathcal{R}^{\prime}} d x \int_{\mathcal{R}^{\prime}} d y W(x ; y)}} .
$$

This completes the definition of general covariant quantum theory.

Whether or not the limit $\mathcal{R} \rightarrow x$ can be taken, as well as any peculiar property of the probabilities in this limit, depends on the dynamics. Notice that the limit of the amplitude $A_{\mathcal{R}, \mathcal{R}^{\prime}}$ as $\mathcal{R}$ shrinks to $x$ and $\mathcal{R}^{\prime}$ to $x^{\prime}$ is proportional to $W\left(x, x^{\prime}\right)$. Therefore $W\left(x, x^{\prime}\right)$ is proportional to the amplitude for the system to be at $x$ if it was at $x^{\prime}$. However, the proportionality factors $C_{\mathcal{R}} C_{\mathcal{R}^{\prime}}$ are dimensional and may diverge in the limit. Furthermore the divergence may depend on the way the limit is taken. For instance, it may depend on the shape of the region approaching the point $x$.

\section{APPLICATIONS}

We now sketch the application of the general formulation discussed above to a number of physical systems, of increasing complexity.

\section{A. Non relativistic particle}

Does the general theory defined in Section III agrees with the conventional quantum theory of a non-relativistic particle studied in Section III? The interpretation of the states $|\mathcal{R}\rangle$ is precisely the same as the one we have derived for the free particle, and therefore is okay. What about the interpretation of $\left\langle\mathcal{R}^{\prime} \mid \mathcal{R}\right\rangle$, for sufficiently small regions, as a transition amplitude? Let us distinguish three cases, according to whether $\mathcal{R}^{\prime}$ is entirely in the past of $\mathcal{R}$, entirely in the future, or neither.

First, we exclude the last case from our considerations, on the ground that the postulated interpretation demands $\mathcal{R}$ and $\mathcal{R}^{\prime}$ to be smaller than any other relevant dimension in the problem. In order for $\mathcal{R}$ and $\mathcal{R}^{\prime}$ not to be time ordered, their relative time localization must be of the same order than their size.

Second, consider the case in which $\mathcal{R}^{\prime}$ is in the future of $\mathcal{R}$. In this case, the amplitude for detecting in $\mathcal{R}^{\prime}$ a particle prepared in $\mathcal{R}$ is indeed proportional to $\left\langle\mathcal{R}^{\prime} \mid \mathcal{R}\right\rangle$, where the proportionality factor depends on the efficiency of the detector. Therefore the interpretation suggested agrees with the prediction of conventional quantum mechanics.

Finally, consider the case in which $\mathcal{R}^{\prime}$ is in the past of $\mathcal{R}$. Strictly speaking, this case refers to a situation which has no meaning in conventional quantum mechanics: it refers to a situation in which the measurement is made at an earlier time than the preparation. Therefore the general theory given in Section III gives more predictions than the ones usually considered in conventional quantum theory. The additional predictions can be more accurately denoted "retrodictions", since they are statements about a time which is in the past with respect to the time at we assume to have information about the state. Jim Hartle has long argued that such retrodictions can be added to standard predictions of quantum theory, and in fact, that they have to be added, if we want to make sense of any statement about the past deriving from our knowledge of the present. Either we give up the possibility of making any statement about the past, or we take retrodictions as these statements. We refer to Hartle's paper [1] for a detailed discussion.

Whatever position we take about retrodiction, the usual predictions of the quantum theory of a non relativistic particle are recovered from the general formalism of Section III. The extension from the free case to the case with a potential is immediate. 


\section{B. Time of arrival}

If the reader is not interested in this problem, this section can be skipped without prejudice for understanding what follows. A simple application of the above considerations is to the problem of the time of arrival in quantum theory. This problem has generated considerable discussions [11. As far as we understand, there is no agreement on its solution. The problem is the following. Suppose that at $T=0$ a nonrelativistic particle is in the state $\Psi(\mathrm{X})$. A particle detector is placed at the origin. At what time $\mathrm{T}$ will the particle detector detect the particle? More precisely, how can we compute the probability distribution in time $\rho(\mathrm{T})$ that the detector detects the particle at time T? Surprsingly, there is no agreement on the solution of this simple problem of non relativistic quantum mechanichs, in spite of the fact that the problem can presumably be experimentally investigated. Different authors have computed different distributions $\rho(\mathrm{T})$ !

The considerations in the previous section suggest the following answer to the problem. First, no detector can be concentrated at the origin. Second, the time resolution of the particle detector can only be finite. Therefore, the only probabilities that we can realistic hope to measure are the probabilities that the particle be measured in a small but finite spatial interval $I$ around the origin, in a small but finite time interval of size $\epsilon$ around the time T. Let us imagine that the particle detector is placed around the origin. It detects the particle in the finite region $I$, and it has a discrete set of pointer variables $i_{n}$. The $n$-th pointer variable $i_{n}$ indicates that the particle has been detected by the apparatus between the times $\mathrm{T}_{n}=n \epsilon$ and $\mathrm{T}_{n+1}=(n+1) \epsilon$. In other words, in spacetime language, we consider a collection of detectors. The $n$-th detector is a spacetime detector of finite resolution $I \times \epsilon$ around the spacetime point $\mathrm{X}=0, \mathrm{~T}=n \epsilon$. We denote this spacetime region as $R_{n}$.

We can then associate a two-state model detector of the kind described in the Section II A to each region $R_{n}$, and the computation of the probabilities for their final configuration at some later time $\mathrm{T}_{f i n}$ is a standard exercize in quantum theory.

The important interpretational point is the following. Does one of the detectors detects whether at the time $\mathrm{T}$ the particle is at the space point $\mathrm{X}$, or does this detector detects whether, at the origin, the passage time of the particle is T? In other words, is this a position measurement at a given time or a time measurement at a given position? Is it a projector on an eigenspace of the position operator $\mathrm{X}(\mathrm{T})$ or of a time of arrival operator $\mathrm{T}(\mathrm{X})$ ? The answer is that a realistic detector is neither of the two. It is an approximation to both quantities. If we take into account that realistic measurements cannot have infinite time resolution, the distinction between measurement of position at a certain time and measurement of time of arrival at a certain position, disappears.

\section{Relativistic particle, I}

The quantum theory of a single relativistic particle is not a realistic theory, since it neglects the physical phenomena of particle creation which are described by quantum field theory. Nevertheless it is interesting to ask whether there exist a logically consistent quantum theory, or several, whose classical limit is the dynamics of a single relativistic particle, and which respects the Lorentz invariance of the classical theory. We discuss two such quantizations. In the first we consider only positive frequency solutions of the Klein Gordon equation, in the second, we consider a theory for both frequencies.

We start with the following covariant formulation of the classical theory. We take $\mathcal{M}$ to be Minkowski space, and the constraint $C$ to be given by the two conditions

$$
\begin{gathered}
p^{2}=m^{2}, \\
E>0 .
\end{gathered}
$$

where $p=(P, E)$ and $p^{2}=-P^{2}+E^{2}$. (We use here $\hbar=c=1$.) Upon quantization the constraint (57) becomes the Klein Gordon equation and positive energy condition (58) becomes the restriction to positive frequencies. Together these constraints restrict the wavefunction to be the Fourier transform of a function supported on the upper mass hyperboliod in momentum space. The corresponding propagator is

$$
W(x, y)=\int \frac{d^{2} p}{2 \pi} \delta\left(p^{2}-m^{2}\right) \theta(E) e^{-i p(x-y)}=\int \frac{d P}{2 \pi} \frac{1}{2 E(P)} e^{i P\left(x^{1}-y^{1}\right)-i E(P)\left(x^{0}-y^{0}\right)},
$$

where $E(P)=+\sqrt{P^{2}+m^{2}}$, and $x=\left(x^{1}, x^{0}\right)=(\mathrm{X}, \mathrm{T})$. It is easiest to represent the Hilbert space in momentum space. Let

$$
\tilde{f}(p)=\int d^{2} x f(x) e^{-i(p \cdot x)}
$$


We have then

$$
(f, g)_{C}=\int \frac{d^{2} p}{2 \pi} \delta\left(p^{2}-m^{2}\right) \theta(E) \tilde{f}(p) \tilde{g}(p) .
$$

Therefore the state space can be represented by $\mathcal{H}=L_{2}\left[R^{2}, \delta\left(p^{2}-m^{2}\right) \theta(E) d^{2} p\right] / \mathcal{I}$, the quotient of the space of functions on $R^{2}$ whose restrictions to the upper mass hyperboloid are square integrable with the Lorentz invariant measure, by the zero norm subspace $\mathcal{I}$ - the subspace of functions which vanish almost everywhere on the upper mass hyperboloid. Clearly the states can be expressed in terms of functions on the upper mass shell only, which (once an inertial frame is chosen) can be written as functions of the spatial momentum $P$ only: The Hilbert space is then $\mathcal{H}=L_{2}\left[R, \frac{d P}{2 E(P)}\right]$, with the wavefunction corresponding to $\tilde{f}$ being $\Psi(P)=\tilde{f}(P, E(P))$ and the inner product given by

$$
(\Psi, \Theta)_{C}=\int \frac{d P}{2 \pi} \frac{1}{2 E(P)} \Psi \overline{(P)} \Theta(P) .
$$

Historically two types of (generalized) states have been associated to spacetime points $x=(\mathrm{X}, \mathrm{T})$ in relativisitic quantum mechanics. First 13] there is the Philips state $\Phi_{x}$, which we also denote $|x\rangle$, which is the spacetime smeared state defined by the spacetime delta function $f(y)=\delta^{2}(y-x)$ :

$$
\Phi_{x}(P)=\langle P \mid x\rangle=e^{-i\left(P x^{1}-E(P) x^{0}\right)} .
$$

Second [14, there is the Newton-Wigner state $\Psi_{x, K}$, which we also denote $|x, K\rangle$ :

$$
\Psi_{x, K}(P)=\langle P \mid x, K\rangle=\sqrt{2 E(P)} e^{-i(P \mathrm{X}-E(P) \mathrm{T})} .
$$

The Philips state depends only the spacetime point $x$, while the Newton-Wigner state depends on $x$, as well as on the choice of an inertial frame $K$, the frame to which the energy $E(P)$ and all other space and time components in the expression (64) are refered. The solutions of the Klein-Gordon equation corresponding to these states are, respectively,

$$
\phi_{x}(y)=W(y, x)
$$

and

$$
\psi_{x, K}(y)=\int d T^{\prime \prime} W\left(y, \mathrm{X}, \mathrm{T}^{\prime \prime}\right) v\left(\mathrm{~T}-\mathrm{T}^{\prime \prime}\right)
$$

where (reinstating dimensionful constants and normalizing the Newton-Wigner state with a factor $\left.\left(m c^{2}\right)^{-1 / 2}\right)$,

$$
v(\mathrm{~T})=\int \frac{d E}{2 \pi \hbar} \sqrt{\frac{2|E|}{m c^{2}}} e^{i E \mathrm{~T} / \hbar}=-\frac{1}{2 \sqrt{\pi} \tau_{c}}\left(\frac{\tau_{c}}{|T|}\right)^{-3 / 2}
$$

with $\tau_{c}=\frac{\hbar}{m c^{2}}$ the "Compton period" of the particle.

It is easy to see that two Newton-Wigner states, corresponding to two distinct points but the same inertial frame and the same time, are orthogonal. Indeed

$$
\left\langle(\mathrm{X}, \mathrm{T}), K \mid\left(\mathrm{X}^{\prime}, \mathrm{T}\right), K\right\rangle=\delta\left(\mathrm{X}, \mathrm{X}^{\prime}\right) .
$$

Furthermore, as we vary $\mathrm{X}$ at fixed $\mathrm{T}$ these states span the Hilbert space, forming a basis. There exists, therefore, a selfadjoint position operator $X_{K}(T)$ diagonal in this basis -

$$
X_{K}(T)|(\mathrm{X}, \mathrm{T}), K\rangle=X|(\mathrm{X}, \mathrm{T}), K\rangle,
$$

- which is called the Newton-Wigner position operator. At $\mathrm{T}=0$ its form in the representation $\Psi(P)$ adapted to the frame $K$ is

$$
X_{K}=i \sqrt{E(P)} \frac{d}{d P} \frac{1}{\sqrt{E(P)}}
$$


The Newton-Wigner states are defined with respect to a particular inertial frame. The state $|(\mathrm{X}, \mathrm{T}), K\rangle$ is not a spatial position eigenstate with respect to the position operator of another frame. Moreover, $|x, K\rangle$ and $\left|x^{\prime}, K\right\rangle$ are not orthogonal unless $x \neq x^{\prime}$ are simultaneous in the frame $K$. It is not sufficient that they be spacelike separated. The Phillips states do not depend on a choice of reference frame and their inner products $\langle x \mid y\rangle=W(x, y)$ show that they are not quite orthogonal for any spacelike separated $x, y$.

Let us now see how these results appear from the point of view of the general theory of Section [II]. Let us consider a small region $\mathcal{R}$ centered in the points $x$, and study the limit of the state $|\mathcal{R}\rangle$ as $R \rightarrow x$. We can take for instance a rectangular region and scale its spacial and temporal sides as $L \rightarrow \epsilon L$ and $T \rightarrow \epsilon T$, adjusting the normalization of the state appropriately. If the normalized characteristic function approximates a 2 dimensional delta function, then the state we obtain approximates the Phillips state of the point, namely $|x\rangle$.

The states $|x, K\rangle$ can also be obtained as limits of characteristic states, using regions of a more complicated shape. For instance, the Newton-Wigner state centered on the spacetime origin can be obtained using the region defined by $|\mathrm{X}|<\epsilon\left(\frac{\tau_{c}}{T}\right)^{3 / 2}$. For small $\epsilon$, we obtain a state that approximates $|(0,0), K\rangle$. Of course, the asymptotic points of this thin diamond shaped region in Minkowski space pick out the axes of a specific Lorentz frame. Hence the frame dependence of the Newton-Wigner states.

Can detectors be built that detect these states? In each Lorentz frame, the theory is just like the non relativistic particle theory with hamiltonian $H=+\sqrt{P^{2}+m^{2}}$. We can therefore use the same detector described for the non relativistic particle, with appropriately shaped interaction regions. In all this, of course, one should keep in mind that the theory is not realistic, since reality is described by quantum field theory. For detectors corresponding to the Newton-Wigner operator, see also [3]. Therefore the two kinds of states correspond simply to the "point-like" limits of two distinct kinds of measurements. Notice that the detectors corresponding to the Newton-Wigner states associated with a single equal time surface collectively measure the Newton-Wigner position, with the eigenvalue obtained being the position of the (only) detector that finds the particle.

Both states propagate faster than light, in the sense that there is a finite probability that the particle be detected at two spacelike separated points. There is nothing logically inconsistent in this: it is simply a prediction from this quantum theory. In the classical limit, the trajectories stay inside the light cone.

Notice that there are two distinct ways of characterizing a state $|\psi\rangle$ by means of a function of the position at fixed time $\mathrm{T}=0$ in a frame $K$. First, we can take $\Phi(\mathrm{X})=\langle x \mid \psi\rangle$ with $x=(\mathrm{X}, 0)$. Second, we can take $\Psi(\mathrm{X})=\langle(\mathrm{X}, 0), K \mid \psi\rangle$. The first is the the value of the solution $\psi(\mathrm{X}, \mathrm{T})$ of the Klein-Gordon equation corresponding to the state at $\mathrm{T}=0$, while the second is the amplitude of finding the particle in $x=(\mathrm{X}, 0)$ by means of a Newton-Wigner position measurement in frame $K$. The two quantities are distinct. Both characterize the state uniquely. They are related by

$$
\begin{aligned}
& \Phi(\mathrm{X})=\int \frac{d P}{2 \pi} \frac{1}{2 E(P)} \Psi(P) e^{i P \mathrm{X}} \\
& \Psi(\mathrm{X})=\int \frac{d P}{2 \pi} \frac{1}{\sqrt{2 E(P)}} \Psi(P) e^{i P \mathrm{X}} .
\end{aligned}
$$

\section{Relativistic particle, II}

A different quantum theory for a single relativistic particle is obtained by dropping the positive frequency condition (58). The Hilbert space $\mathcal{H}$ is then formed by functions with support on both hyperboloids. The propagators is

$$
\begin{aligned}
W(x, y) & =\int \frac{d^{2} p}{2 \pi} \delta\left(p^{2}-m^{2}\right) e^{i p(x-y)} \\
& =\int \frac{d P}{2 \pi} \frac{1}{2 E(P)}\left(e^{i P\left(x^{1}-y^{1}\right)-i E(P)\left(x^{0}-y^{0}\right)}+e^{-i P\left(x^{1}-y^{1}\right)+i E(P)\left(x^{0}-y^{0}\right)}\right) .
\end{aligned}
$$

Again, this is proportional to the probability amplitude of finding the particle in $x$ if it was in $y$. The key difference between this theory and the one in the previous subsection is the fact that in this theory the spatial localization of the particle in a given frame is not sufficient to determine the state. Indeed, a function $\psi(p)$ on both hyperboloids is not determined by a single function of the spatial momentum, but rather by two functions of momentum:

$$
\Psi_{ \pm}(P) \equiv \psi(P, \pm E(P)) .
$$

We can still define the Newton-Wigner position operator at $\mathrm{T}=0$ in a given reference frame 


$$
X \Psi_{ \pm}(P)=-i \hbar \sqrt{E(P)} \frac{d}{d P} \frac{1}{\sqrt{E(P)}} \Psi_{ \pm}(P)
$$

but this operator no longer constitutes a complete set of commuting observables. Its (generalized) eigenspaces are doubly degenerate. They include a positive frequency and a negative frequency component. Accordingly, the position of the particle at $\mathrm{T}=0$ does not determine the state uniquely.

In a sense, the theory describes a particle that can exist in two states: either as a particle or as an antiparticle. The dynamics does not mix the the two, but a measurement does. Different kinds of measurements can select different mixtures of positive and negative frequency states.

A measurement that checks whether the particle is in a small region $\mathcal{R}$ defines the state $|\mathcal{R}\rangle$, represented by the (non-normalized) solution of the Klein-Gordon equation

$$
\psi_{\mathcal{R}}(x)=\int_{\mathcal{R}} W(x, y) d y
$$

If a particle is in such a state, the probability amplitude of finding it in a small region $\mathcal{R}^{\prime}$ is given by (56).

\section{E. Quantum particle in a curved spacetime}

Let $g_{\mu \nu}(x)$ be a globally hyperbolic spacetime metric. Can we define a quantum theory of a single particle moving in the spacetime defined by this metric? The quantization of Section IV cannot be generalized to a curved spacetime because in general there is no split of the space of the solutions of the curved space Klein-Gordon equation into positive and negative frequencies. However, the quantization of Section IVD can. The classical particle is characterized by the constraint

$$
p^{2} \equiv g^{\mu \nu}(x) p_{\mu} p_{\nu}=m^{2} .
$$

where $p=(P, E)=\left(p_{1}, p_{0}\right)$. The quantum constraint becomes the curved spacetime Klein-Gordon equation

$$
C \psi(x)=\left(g^{\mu \nu}(x) D_{\mu} D_{\nu}-m^{2}\right) \psi(x)=0 .
$$

where $D_{\mu}$ is the covariant derivative of $g$. The state space if formed by solutions of this equation, and the propagator $W(x, y)$ is defined by

$$
\begin{aligned}
\left(f, f^{\prime}\right)_{C} & =\int d \tau \int d^{2} x \overline{f(x)}\left[e^{i \tau C} f\right](x) \\
& =\int d^{2} x d^{2} y \overline{f(x)} W(x, y) f(y)
\end{aligned}
$$

assuming that the integral in the first line converges.

As before, a measurement that checks whether the particle is in a small spavcetime region $\mathcal{R}$ defines the state $|\mathcal{R}\rangle$, represented by the solution of the Klein-Gordon equation

$$
\psi_{\mathcal{R}}(x)=\int_{\mathcal{R}} W(x, y) d y
$$

If a particle is in such a state, the probability amplitude of finding it in a small region $\mathcal{R}^{\prime}$ is given as before by (56).

\section{F. Quantum cosmology}

We do not consider here the problem of making sense of the quantum theory of a single universe, in which the frequency interpretation of probabilities is questionable, and in which the notion of external observer, required by the Copenhagen interpretation, is of difficult use. Instead, we assume that the Wheeler DeWitt equation considered does not describe all degrees of freedom of the universe, but only a subset of these (say the gravitational ones, or just some cosmological variables), so that we can still assume, for the sake of the interpretation, that other degrees of freedom in the universe are treated classically, and can be used to define a classical Copenhagen external observer. And also that the dynamics that we are studying is such that in some appropriate sense measurements could be repeated on 
same states, and thus the frequency interpretation of probability could be used. Whether or not these assumptions are physically viable is a problem we do not address here. We focus only on the issue of time.

We assume we are given a Wheeler DeWitt equation, of the form $C \psi=0$ as a differential equation for a wave function $\psi(x)$, where $x \in \mathcal{M}$ represents a set of physical variables. The space $\mathcal{M}$ can be infinite dimensional, for instance the space of the three geometries, or finite dimensional. For instance, in a simple homogeneous isotropic cosmological model with a scalar field, we have $x=(a, \phi)$, where $a$ is the radius of the universe and $\phi$ is the spatially constant value of the scalar field. We focus here on the finite dimensional case, since we are interested in the conceptual issue of time only, leaving the generalization to an infinite dimensional $\mathcal{M}$ to further developments. We also assume that we can fix a measure $d x$ on $\mathcal{M}$ giving an auxiliary Hilbert space $H_{\text {aux }}=L_{2}[\mathcal{M}, d x]$ in which $C$ is self adjoint, and a space $\Phi$ formed by smooth compact support functions $f(x)$ on $M$. The question is whether we can give a consistent probabilistic interpretation to the solutions of the Wheeler DeWitt equation without selecting in $\mathcal{M}$ a time variable, or a preferred time direction.

Our strategy should be clear at this point. We define the bilinear form ( , $)_{C}$ on $\Phi$ in terms of Equation (48). We mod out by the zero norm states and complete in norm, obtaining an Hilbert space $\mathcal{H}$. This can be identified as a space of solutions of the Wheeler DeWitt equations. Any function $f$ in $\Phi$ defines a state $|f\rangle$ of the system. In particular, for any small but finite region $\mathcal{R}$ in $\mathcal{M}$ we consider the state $|\mathcal{R}\rangle$ and we give it the physical interpretation of a state which has been found in the region $\mathcal{R}$ of the extended configuration space. The probability to find the system in the region $\mathcal{R}^{\prime}$ is then given by Eq. (55).

\section{G. Quantum gravity}

In reference [15], a strategy for computing transition amplitudes $W\left(s, s^{\prime}\right)$ between (generalized) three geometries $s, s^{\prime}$ is given. The space on which the generalized three geometries $s$ live is a collection of finite dimensional components and carries a natural measure $d s$. We can therefore interpret the theory along the lines discussed here. The theory defined probabilities of finding a certain three geometry (with matter), within a small error, after a certain three geometry (with matter), within a small error, has been detected.

\section{SOME CONCEPTUAL ISSUES}

The introduction of the spacetime-smeared observables raises certain general issues, which we discuss here.

\section{A. Probability of what?}

Consider the following objection

- The probability of finding the particle in the space interval $I$ at $\mathrm{T}=0$ is meaningful because the alternative is well defined: it is the probability that the particle be elsewhere, namely in $\mathbb{R}-I, \mathbb{R}$ being the $\mathrm{T}=0$ real line. But the probability of the measurement outcome of finding the particle in an arbitrary spacetime region $\mathcal{R}$ has no meaning, because the set of alternatives is not defined.

Consider a measurement of position at fixed time $T$, on an assigned initial state $\Psi$, and assume here that we can perform the measurement with infinite time resolution. Let us say that the probability that particle is in the interval $I$ is $P$. This probability can be measured (to a given accuracy) by repeated measurements, as relative frequency of outcomes. If we talk about frequency, we have to specify the set of alternatives out of which the outcome is considered. Otherwise, the notions of probability or frequency do not make sense.

In a measurement of position, the alternative is often taken to be that the particle is elsewhere (not in $I$ ) at $\mathrm{T}=0$. Thus, $P$ is interpreted as the probability that the particle is $I$, as opposite of being in $\mathbb{R}-I$. To make sense of this definition of alternatives, one should assume that we have an infinity of detectors spread all along the real line $\mathrm{T}=0$, all the way to infinity: some detectors on Andromeda, some on Orion, and others further away. There is nothing wrong in idealizations, but is this an useful one? Is this idealization needed to make sense of the measurement of the localization of a particle? In a concrete experiment, what we do is simply to turn on the detector, and see whether it has detected the particle. Why should this be related to the behavior of another ideal particle detector on Andromeda?

It is more reasonable to assume that the alternatives we consider are whether this particular detector has detected the particle or not. We can consider a set of two alternatives only: one is that the detector detects the particle, the 
other is that the detector does not detect the particle. Once the initial state of the particle is determined and the detector is specified, these two alternatives are well defined and form a complete set of alternatives. The frequency interpretation of measurement positions of quantum particles in a real experiment, for instance, is clearly this one, and not whether the particle is in $I$ as opposite at being somewhere else.

In the case of a non relativistic particle, the theory tells us that if the particle is detected in $I$, then it cannot be detected in $\mathbb{R}-I$. This, however is a consequence of the dynamics of the theory, not an a priori requirement needed to make sense of the measurement.

Consider now a small spacetime region $\mathcal{R}$ and a detector there. We can consistently define the probability that the particle is in $\mathcal{R}$ as the probability that the detector detects the particle. This is not the probability that the particle is $\mathcal{R}$ as opposite of being somewhere else. It is the probability that the particle be detected, as opposite of not being detected.

\title{
B. What is an observable? Partial observables and complete observables
}

\author{
Consider the following objection
}

- In quantum mechanics, the position of the particle $\mathrm{X}$ is an observable, while the time $\mathrm{T}$ is an external parameter. One should not confuse the two, which are very distinct. In particular, if we say that we measure whether the particle is in $\mathcal{R}$, we are assuming that we can measure quantum mechanically both position and time, and this is a mistake, because time is not an observable.

This point is discussed in detail in reference [16]. Here we give a short account of the response, referring the reader to [16] for more details. There is a certain ambiguity in the notion of observable. This ambiguity is reflected for instance in the difference between the quantum theory observables in the Shrödinger picture (the "position" operator $\mathrm{X}$ ) and in the Heisenberg picture (the "position at time T" operator $\mathrm{X}(\mathrm{T})$.) To disentangle this ambiguity, let us start from the classical mechanics of a single particle. At every time $\mathrm{T}$, we can measure the position $\mathrm{X}$ where the particle is. Let us use the expression "complete observable" to indicate the position of the particle at a given time. Thus, a complete observable is for instance the position at $\mathrm{T}=0$, and a distinct complete observable is the position at $\mathrm{T}=3$ seconds. We use the expression "partial observable" to generically indicate the "position" or the "time". More precisely, we operationally define a partial observable as any measurement procedure that produces a number (checking where is the particle, looking at the clock...). We define a complete observable as a measurements procedure that gives a number that can be predicted from the knowledge of the state of motion of the system (or, in quantum theory, whose probability distribution can be predicted. A typical complete observable is formed by the conjunction of two (or more) partial observables.

In non-covariant theories, partial observables fall in two distinct groups: independent and dependent ones. Independent partial observables characterize the spacetime position where the measurement happens. Thus, time is the independent partial observable in the mechanics of a nonrelativistic particle, while the position of the particle is the dependent one. In Maxwell theory, time and position are two independent partial observables, the electric and magnetic field are the dependent observables, and a complete observable is given by the value of the field at a given time and a given position.

The novelty introduced with general relativity, and the peculiarity of all covariant theories is precisely the fact that the a priori distinction between dependent and independent partial observables is lost. Complete observables are still given by conjunctions of partial observables, but dependent and independent partial observables are not distinguished. Thus for instance in the cosmology of a isotropic universe with a constant scalar field, $a$ and $\phi$ are both partial observables, but they are on an equal footing.

Back to the objection, the truly observable quantity is the relation between $\mathrm{X}$ and $\mathrm{T}$. That is, a state of motion determines a unique relation between $\mathrm{X}$ and $\mathrm{T}$. Because of the specific form of the dynamics of the system, we can then treat the two quantity dissymmetrically. We can treat $\mathrm{T}$ as an external independent parameter and $\mathrm{X}$ as a dynamical variable. In a general case, however, this may be impossible. A general state of motion will determine a relation between a set of variables in an extended configuration space $\mathcal{M}$. Thus, the distinction a priori between $\mathrm{X}$ and $\mathrm{T}$ mentioned in the objection is viable, but not necessary, in a non covariant theory. Is not anymore viable in a covariant context.

\section{Repeated measurements}

Up to now, we have considered the situation in which quantum theory is employed to predict the probability for the outcome of a measurement on a state prepared in another measurement. Standard quantum theory, however, has 
a wider application: it can be applied to repeated measurements. That is, quantum theory addresses the following problem. Assume we know that the system is in a state $|\Psi\rangle$; let a first measurement be performed and let us assume that we know the outcome $A$ of the measurement. What is the state after the measurement? Equivalently, how can we compute the probability $P$ of obtaining $A$ and obtaining $B$ in a another measurement (of a different observable)?

The standard answer is the following. If $\Pi_{A}$ is the projector on the eigenspace corresponding to the outcome $A$, and $\Pi_{B}$ is the projector on the eigenspace corresponding to the outcome $B$, then the probability of obtaining $A$ and $B$ is $P_{B A}=\left.\left|\Pi_{B} \Pi_{A} \Psi\right\rangle\right|^{2}$ if the $A$ measurement is the first in time. It is $P_{A B}=\left.\left|\Pi_{A} \Pi_{B} \Psi\right\rangle\right|^{2}$ if the $B$ measurement is the first in time. In general, the two projectors $\Pi_{A}$ and $\Pi_{B}$ do not commute, and therefore $P_{A B}$ is not equal to $P_{B A}$. Therefore the probability of a set of outcomes is determined by the time ordering of the measurements.

If we try to analyze this situation in our covariant framework we find that the framework it is not complete, since time ordering is not defined in a covariant theory. This fact raises a difficulty. To see this, consider three regions of the extended configuration space: $\mathcal{R}, \mathcal{R}^{\prime}$ and $\mathcal{R}^{\prime \prime}$. Let the state be $|\mathcal{R}\rangle$. What is the probability that the system is detected in $\mathcal{R}^{\prime}$ and in $\mathcal{R}^{\prime \prime}$ ?

It is tempting to say that the measurement of the system in $\mathcal{R}^{\prime}$ prepares a state $\left|\mathcal{R}^{\prime}\right\rangle\left\langle\mathcal{R}^{\prime} \mid \mathcal{R}\right\rangle \equiv \Pi_{\mathcal{R}^{\prime}} \mid \mathcal{R}>$ on which the $\mathcal{R}$ " measurement acts and so the probability is $\| \Pi_{\mathcal{R}^{\prime \prime}} \Pi_{\mathcal{R}^{\prime}} \mathcal{R}>\left.\right|^{2}$. But which projector, $\Pi_{\mathcal{R}^{\prime}}$ or $\Pi_{\mathcal{R}^{\prime}}$, should be applied first?

In the case of a non-relativistic particle being detected in two very small spacetime regions $\mathcal{R}^{\prime}$ and $\mathcal{R}$ " with $\mathcal{R}$ " to the future of $\mathcal{R}^{\prime}$ and both to the future of $\mathcal{R}$, the formula $P=|| \Pi_{\mathcal{R}^{\prime \prime}} \Pi_{\mathcal{R}^{\prime}} \mathcal{R}>\left.\right|^{2}$ does indeed reproduce the correct standard result. On the other hand, the quantity $P=\left.\left|\Pi_{\mathcal{R}^{\prime}} \Pi_{\mathcal{R}}, R\right\rangle\right|^{2}$ does not seem to have any clear physical meaning in the theory [18]. But if it is so, how can we generalize this result to an arbitrary configuration space $\mathcal{M}$, in which no time ordering is defined? The projectors act on the wavefunctions in all of spacetime, including the past, so it is not obvious that the projector corresponding to the later measurement should be put on the left. Indeed it may not be possible to define which region is later. For instance in a special relativistic context even disjoint regions may be partly in the future and partly in the past of each other.

There are two, possibly related approaches. In the first approach [17] the series of measurements is treated as a single measurement by including part of the measurement aparatus in the system of observation, with the reading of the results of the sequence of measurements by the observer constituting the final, single act of measurement.

For instance, in the example above assume that in the region $\mathcal{R}^{\prime}$ the particle interacts with a two state system $S_{1}$ and in the region $\mathcal{R}^{\prime \prime}$ with a two state system $S_{2}$, as in section IIA. The extended configuration space $\mathcal{M}$ is the physical spacetime $M$ times $\{0,1\}$ times $\{0,1\}$. Assume the initial state is characterized by the region $(R, 0,0)$ of $\mathcal{M}$. Let us ask what is the probability that the system is found in $\left(R_{\mathrm{T}}, 1,1\right)$, where $R_{\mathrm{T}}$ is the $\mathrm{T}=$ constant line in spacetime and $\mathrm{T}$ is later than $\mathcal{R}^{\prime}$ and $\mathcal{R}^{\prime \prime}$. It is then easy to see that the resulting probability amplitude is proportional to $A=\left.\left|T\left(\Pi_{\mathcal{R}^{\prime}} \Pi_{\mathcal{R}^{\prime \prime}}\right) R\right\rangle\right|^{2}$, where $T$ indicates time ordering. That is, time ordering is not produced by an additional postulate of the quantum theory, but simply by the dynamics itself. The quantum theory predicts only outcomes of individual measurements. A sequence of measurements can be reformulated as a single measurement, by including into the system the dynamics of the measurement apparatus.

The second answer is more speculative. In order to use quantum theory, we ideally separate the world into two components. The first component is the system studied, which we denote $S$. The second component is the "observer", namely the rest of the world, which we denote $O$. We think that this separation is intrinsic in the quantum description of the world: quantum theory is always a theory of the interactions of a system $(S)$ over another system $(O)$. 2 On the other hand, the division is arbitrary: indeed, the most remarkable feature of quantum theory is that the descriptions obtained by breaking the world into $S / O$ components in different manners are all consistent with each other [19]. Now, the $S$ system may be a covariant system in which time ordering is not defined. Nevertheless, a time ordering may be introduced by $O$. In other words, the time ordering that selects the relevant probabilities may be the one of the observer, not the one of the system. For instance, imagine that $S$ is formed by the $a$ and $\phi$ degrees of freedom in a cosmological situation. We can take then as $O$ a set of variables describing physics on, say, the Earth, for which a specific time ordering is somehow physically determined. A sequence of measurements in the $\mathcal{M}$ extended configuration space with coordinates $a$ and $\phi$ is then time ordered by the order under which the system $O$ comes in relation with these regions.

\footnotetext{
${ }^{2}$ However, there are well developed attempts to make sense of the quantum theory of "closed" systems, namely systems that do not interact with an external observer. See in particular [1].
} 


\section{CONCLUSION}

We have explored the possibility of defining quantum theory in a covariant form. That is, in a form that allows the independent time variable to be treated on an equal footing with the dynamical variables in the extended configuration space. We expect that such a form of quantum theory is required for understanding the quantum behavior of the covariant systems such as the relativistic gravitational systems.

We have found that much of the peculiar role that the time variable assumes in the conventional formulation of quantum theory is not intrinsic in the quantum behavior of the physical systems, but rather it depends on an idealization of the measurements: the unrealistic assumption that physical measurements could be performed instantaneously. This idealization simplifies the formalism of quantum theory; however, it hides the beautiful symmetry among all variables of the extended configuration space. This symmetry is present in classical mechanics, where it is made manifest by formulations such as the Hamilton-Jacobi theory, or the covariant (presymplectic, parametrized) hamiltonian formulation. The thesis of this paper is that this symmetry is not broken by the physical quantum phenomenology, but only by the unrealistic idealization of instantaneous measurements. Giving up this idealization reveals the same symmetry in the quantum world, opening the way to a formulation of quantum theory sufficiently general to deal with covariant theories.

The technical ingredient to be added to the quantum formalism is the notion of spacetime-smeared quantum state. This is a state generated by a measurements that is not instantaneous. In particular, localization measurements can be naturally described in terms of states associated to spacetime regions, or, more in general, regions in the extended configuration space. The key element of the theory, from this point of view, is the propagator $W(x, y)$. This quantity is a two point function on configuration space, it is closely related (but not identical!) to the "probability amplitude for the system to be detected in $x$ if it was detected in $y$ ". Furthermore, it defines the Hilbert space of the theory, since it is the kernel of the scalar product between spacetime-smeared states, and it defined the "projection" from the space of test functions over configuration space to the Hilbert space itself. In general, $W(x, y)$ is defined by the Wheeled DeWitt operator. In the classical limit, it is easy to see that $W(x, y)$ reduces to the exponential of the classical action, or, more precisely, to a general solution of the Hamilton-Jacobi equation of the system.

The formulation we have suggested makes sense for non relativistic systems, at the (interesting) price of adding the "retrodictions" to the predictions of the quantum theory. It makes sense in the context of (unrealistic) relativistic theories of a single particle, where it helps clarifying the distinction between the different kind of states associated to spacetime points (such as the Newton-Wigner and the Phillips states). It allows us to make sense of the (unrealistic) quantum theory of a single particle in a curved spacetime. It allows us to give a logically consistent interpretation to the (realistic?) quantum cosmological models, as far as the "problem of time" of these models is concerned. We expect also that this general formulation can be taken as a reference scheme in quantum gravity.

The remaining conceptual difficulty regards the possibility of associating probabilities to sequences of measurements. We see two possible solutions to this difficulty. The first by reducing any such sequence to a single measurement or, equivalently, to sets of commuting measurements, by including the apparatus in the theory. The second by introducing the notion of time ordering of the observer.

We thank Rafael Porto and Don Marolf for valuable exchanges on the subject, Bruno Iochum and Raphael Zentner for numerical help with an integral. This work was partially supported by NSF Grants PHY-9900791.

Note added: In the recent paper [20], the interpretation postulate presented here is shown to be correct in the context of the dynamics of a relativistic particle, provided that a Lorentz invariant description of the measurement is utilized.

[1] J Hartle, "Spacetime Quantum mechanics and the quantum mechanics of spacetime", in Gravitation et Quantification: Les Houches, Session LVII, 1992, B Julia, J Zinn-Justin eds (Elsevier Science BV 1995)

[2] RJ Micanek, JB Hartle, Nearly Instantaneous Alternatives in Quantum Mechanics, quant-ph/9602023.

[3] D Marolf, Quantum Observables and Recollapsing Dynamics, Class Quantum Grav 12 (1995) 1199-1220. D Marolf, Group Averaging and Refined Algebraic Quantization: Where are we now?, gr-qc/0011112; D Giulini, D Marolf, A Uniqueness Theorem for Constraint Quantization, gr-qc/9902045.

[4] D Marolf, Models of particle detection in regions of spacetime, Phys Rev A 50 (1994) 939. J Hartle, Phys Rev D44 (1991) 3173. N Yamada, S Takagi, Progr Theor Phys 85 (1991) 985. 
[5] B DeWitt in "Gravitation: An Introduction to Current Research", L Witten ed (John Wiley and Sons: New York 1962), in "Relativity, Groups, and Topology II: Les Houches 1983" B DeWitt, R Stora eds (North-Holland: New York 1984)

[6] CJ Isham, Canonical Quantum Gravity and the Problem of Time, in "Integrable Systems, Quantum Groups, and Quantum Field Theories", LA Ibort, MA Rodriguez eds (Kluwer, London, 1993).

[7] J Klauder, Product Representations and the Quantization of Constrained Systems, quant-ph/9811051, Coherent State Path Integrals at (Nearly) 40 quant-ph/9810043.

[8] JJ Halliwell, J Thorwart, Decoherent histories analysis of the relativistic particle, Phys Rev D64 (2001) 124018. JJ Halliwell, Life in an Energy Eigenstate: Decoherent Histories Analysis of a Model Timeless Universe, gr-qc/0201070.

[9] R Gambini, RA Porto Relational time in generally covariant quantum systems: four models, Phys Rev D63 (2001) 105014; Relational Reality in Relativistic Quantum Mechanics, quant-ph/0105146.

[10] M Reisenberger, C Rovelli, "Sum over Surfaces" form of Loop Quantum Gravity, Phys Rev D56 (1997) 3490-3508. A Perez, C Rovelli, Observables in quantum gravity, gr-qc/0104034. L Crane, A Perez, C Rovelli A finiteness proof for the Lorentzian state sum spinfoam model for quantum general relativity, to appear on Phys Rev Lett.

[11] Y Aharonov, D Bohm, Time in the Quantum Theory and the Uncertainty Relation for Time and Energy, Phys Rev 122 (1961) 1649. DM Rosenbaum, Super Hilbert Space and the Quantum Mechanical Time Operators, J Math Phys 10 (1969) 1127. VS Olkhovsky, E Recami, AJ Gerasimchuk, Time Operator in Quantum Mechanics, Nuovo Cimento 22 (1974) 263. P Busch, M Grabowski, PJ Lathi, Time Observables in Quantum Theory, Phys Lett A 191 (1994) 357. P Blanchard, A Jadczyk, Time of Events in Quantum Theory, Helv Phys Acta 69 (1996) 613-635. N Grot, C Rovelli, RS Tate, Time-ofarrival in quantum mechanics, Phys Rev A 54 (1996) 4679. J Leon, Time-of-arrival formalism for the relativistic particle. J Phys A30 (1997) 4791.

[12] C Rovelli, What is observable in classical and quantum gravity?, Classical and Quantum Gravity 8, 297 (1991), Quantum reference systems, Classical and Quantum Gravity 8, 317 (1991), Quantum mechanics without time: a model, Physical Review D42, 2638 (1991), Time in quantum gravity: an hypothesis, Physical Review D43, 442 (1991).

[13] TO Philips, Phys Rev 136 (1964) B893.

[14] T Newton, E Wigner, Rev Mod Phys 21 (1949) 400.

[15] A Perez, C Rovelli, Observables in Quantum Gravity, gr-qc/0104034

[16] C Rovelli, Partial observables, gr-qc/0110003. See also Is there incompatibility between the ways time is treated in general relativity and in standard quantum mechanics?, in "Conceptual problems of quantum gravity", A Ashtekar, J Stachel eds (Birkhauser, New York, 1991).

[17] D Marolf, private communication.

[18] J Hartle, Time and Time Functions in Parametrized Non-Relativistic Quantum Mechanics, Class Quant Grav 13 (1996) 361.

[19] C Rovelli, Relational Quantum Mechanics, International Journal of Theoretical Physics, 35, 1637 (1996).

[20] C Rovelli, D Marolf, Relativistic Quantum Measurement, gr-qc/0203056. 